ARTICLE

httpst//doi.org/10.1038/s41467-019-11559-1

\title{
Mapping histone modifications in low cell number and single cells using antibody-guided chromatin tagmentation (ACT-seq)
}

Benjamin Carter (10 ${ }^{1,2}$, Wai Lim Ku (1) ${ }^{1,2}$, Jee Youn Kang ${ }^{1}$, Gangqing Hu${ }^{1}$, Jonathan Perrie ${ }^{1}$, Qingsong Tang $^{1}$ \& Keji Zhao (iD ${ }^{1}$

Modern next-generation sequencing-based methods have empowered researchers to assay the epigenetic states of individual cells. Existing techniques for profiling epigenetic marks in single cells often require the use and optimization of time-intensive procedures such as drop fluidics, chromatin fragmentation, and end repair. Here we describe ACT-seq, a streamlined method for mapping genome-wide distributions of histone tail modifications, histone variants, and chromatin-binding proteins in a small number of or single cells. ACT-seq utilizes a fusion of Tn5 transposase to Protein A that is targeted to chromatin by a specific antibody, allowing chromatin fragmentation and sequence tag insertion specifically at genomic sites presenting the relevant antigen. The Tn5 transposase enables the use of an index multiplexing strategy (iACT-seq), which enables construction of thousands of single-cell libraries in one day by a single researcher without the need for drop-based fluidics or visual sorting. We conclude that ACT-seq present an attractive alternative to existing techniques for mapping epigenetic marks in single cells.

\footnotetext{
${ }^{1}$ Laboratory of Epigenome Biology, Systems Biology Center, National Heart, Lung and Blood Institute, NIH, Bethesda, MD, USA. ${ }^{2}$ These authors contributed equally: Benjamin Carter, Wai Lim Ku. Correspondence and requests for materials should be addressed to K.Z. (email: zhaok@nhlbi.nih.gov)
} 
T echniques for mapping epigenetic states in individual cells have enhanced our understanding of differentiation and cell-to-cell variation. Multiple single-cell approaches have been developed to map transcriptomes and chromatin organization, and these methods are proving invaluable in fields such as cancer research ${ }^{1,2}$. Comparatively few methods exist for singlecell profiling of histone tail modifications $s^{1,3}$, which play important roles in epigenetic control of gene expression and development ${ }^{4}$. Here we describe antibody-guided chromatin tagmentation sequencing (ACT-seq), a technique to assay distributions of epigenetic marks in a small number of cells or thousands of single cells simultaneously.

ACT-seq utilizes Tn5 transposase, which is commonly used to map chromatin accessibility and structure ${ }^{5-7}$. We fused the $\mathrm{N}$ terminus of Tn5 transposase to Protein A (PA) to form a fusion protein hereafter referred to as PA-Tnp (Supplementary Fig. 1). The PA domain of the fusion protein is first bound to an antibody that is selected to target an epigenetic mark or chromatin-bound protein of interest. The complex is then incubated with permeabilized cells and is guided to chromatin by the associated antibody. After washing away unbound complex, the transposition reaction is initiated by addition of an $\mathrm{MgCl}_{2}$-containing buffer, which results in insertion of sequence tags at sites of bound PA-Tnp. The reaction is terminated by incubation with EDTA and proteinase. The labeled fragments are directly amplified using PCR and sequenced using Illumina HiSeq technology.

\section{Results}

ACT-seq robustly maps epigenetic marks in bulk-cell samples. To evaluate the efficiency of ACT-seq, we mapped the distributions of a variety of epigenetic features in HEK293T cells: the histone tail modifications $\mathrm{H} 3 \mathrm{~K} 4 \mathrm{me} 1, \mathrm{H} 3 \mathrm{~K} 4 \mathrm{me} 2, \mathrm{H} 3 \mathrm{~K} 4 \mathrm{me} 3$, and $\mathrm{H} 2 \mathrm{~K} 27 \mathrm{ac}$; the histone variant H2A.Z; and the chromatin-binding protein Brd4. Visual inspection using a genome browser revealed highly similar distributions of enrichment in the bulk-cell ACTseq data relative to published ChIP-seq data sets (Fig. 1a) ${ }^{8,9}$. By comparison, little to no specific enrichment was apparent in the ACT-seq mock IgG sample, indicating that the observed signals were antibody-specific. Analysis of statistically significant peaks of enrichment also revealed strong correlations between the data sets obtained using ACT-seq and ChIP-seq (Supplementary Fig. 2). Further, we detected strong average enrichment of H3K27ac, H2A.Z, and Brd4 at transcription start sites (TSS) and enhancer regions in our ACT-seq data (Fig. 1b, c) in agreement with published studies on these factors ${ }^{10-12}$. To determine whether the ACT-seq signal was distinguishable from nonspecific Tn5 activity at regions of open chromatin, we statistically compared the H3K4me3 ACT-seq peaks with a published ATAC-seq data set that was generated using the same cell type ${ }^{13}$. We found that over 11,000 ACT-seq peaks remained after filtering using a false discovery rate threshold of 0.05 , indicating that ACT-seq peaks predominantly arise from antibody binding as opposed to nonspecific Tn5 activity (Supplementary Fig. 3). Taken together, these analyses confirm that ACT-seq and ChIP-seq provide comparable information on enrichment of epigenetic marks in bulk-cell samples.

To evaluate the sensitivity of ACT-seq, we replicated the H3K4me3 data set using samples comprising varying cell numbers. Visual inspection of these data sets revealed that peaks were reliably detected using as few as 1000 cells (Fig. 1d). This also held true for the pattern of average $\mathrm{H} 3 \mathrm{~K} 4 \mathrm{me} 3$ enrichment at the TSS of genes (Fig. 1e). Consistent with these results, we observed strong correlation between statistically significant regions of enrichment in the $\mathrm{H} 3 \mathrm{~K} 4 \mathrm{me} 3 \mathrm{ChIP}$-seq data set and in each of the ACT-seq data sets obtained using different numbers of cells (Supplementary Fig. 4). These results support the reproducibility of ACT-seq data obtained from bulk samples of as few as 1000 cells.

iACT-seq robustly maps epigenetic marks in single cells. To map epigenetic marks in single cells, we adapted the ACT-seq method described in the section above using a barcode multiplexing strategy similar to previously published approaches ${ }^{14,15}$. Permeabilized cells were divided volumetrically into 96 wells at a density of 5000 cells per well. Each well was treated with a separate PA-Tnp complex carrying a unique combination of $5^{\prime}$ and $3^{\prime}$ sequence barcodes. After washing away any unbound complex, the cells were pooled and distributed into a second 96well plate at a density of 18 cells per well using FACS sorting. The transposition reaction was initiated by addition of $\mathrm{MgCl}_{2}$ and terminated by addition of EDTA and proteinase. Library construction and amplification were performed separately in each well using a second set of distinct index barcodes that were unique to each well. The samples were then pooled, purified, and sequenced.

Using this indexing ACT-seq (iACT-seq) strategy, we mapped H3K4me3 enrichment in 1246 individual cells (Fig. 2) and obtained about 2500 unique reads per cell. Visual inspection of the mapped single-cell reads revealed that the read density predominantly clustered in the peaks of enrichment present in the bulk-cell ACT-seq and ChIP-seq data sets (Fig. 2a). Further, the pattern of average enrichment at the TSS regions of annotated genes was highly reproducible among the individual cells (Fig. 2b). Calculations of the precision and sensitivity metrics (Fig. 2c, d) for iACT-seq compare favorably to existing techniques. For example, our analysis found that iACT-seq yielded an average sensitivity of 0.05 and a precision of 0.6 , whereas Drop-ChIP 16 yielded values of 0.07 and 0.53 , respectively. Visual inspection revealed that the precision of iACT-seq also compared favorably to ChIL-seq ${ }^{17}$ (Supplementary Fig. 5). We next examined whether the distribution of $\mathrm{H} 3 \mathrm{~K} 4 \mathrm{me} 3$ enrichment was similar between iACT-seq and ChIP-seq. We found that peaks of statistically significant $\mathrm{H} 3 \mathrm{~K} 4 \mathrm{me} 3$ enrichment were highly correlated between the iACT-seq data and the ENCODE ChIP-seq data set (Fig. 2e, f), in a similar manner to our analysis of the bulk-cell ACT-seq results (Supplementary Fig. 4). Based on our analyses, we conclude that iACT-seq is capable of efficiently mapping epigenetic marks in thousands of individual cells simultaneously, which takes 1 day of bench work.

\section{Discussion}

ACT-seq presents several advantages over many existing methods for profiling epigenetic marks. First, it eliminates procedures that often require laborious optimization or time-consuming incubations such as chromatin fragmentation using sonication or enzymatic digestion, immunoprecipitation, end repair, and adapter ligation. Further, split-pool barcoding can be used in parallel with this method to efficiently profile epigenetic marks in thousands of single cells simultaneously.

Based on our analyses, we found that ACT-seq was capable of robustly mapping epigenomic features in bulk-cell samples including histone tail modifications, histone variants, and chromatin-binding proteins. The iACT-seq method for profiling single cells efficiently reproduced the patterns of enrichment observed in the bulk-cell samples. A simulation was used to calculate the theoretical number of cell doublets (two cells mistakenly identified as a single cell) remaining in the iACT-seq data after filtering (Supplementary Software). Based on this simulation, we estimated that 54 cell doublets remained out of 1246 cells 
a

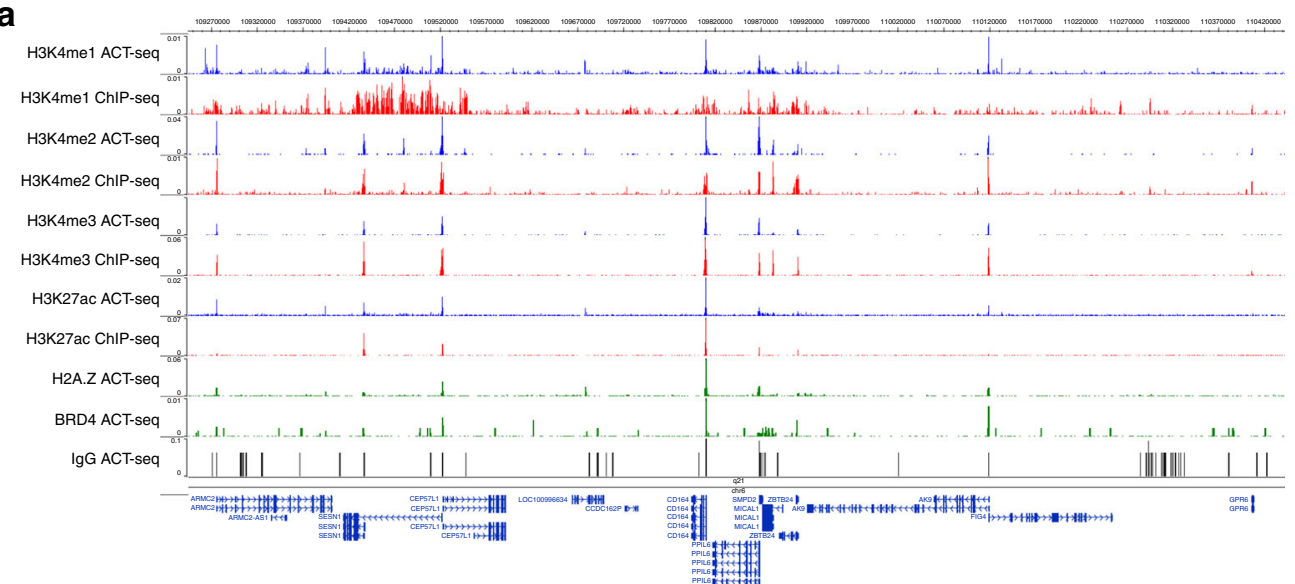

d

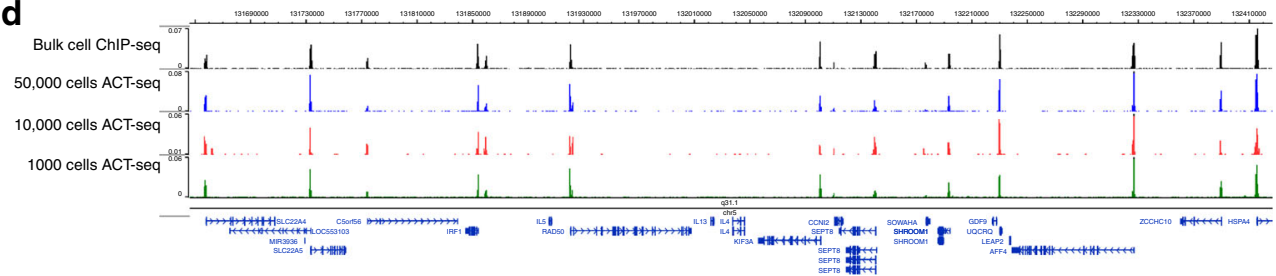

b

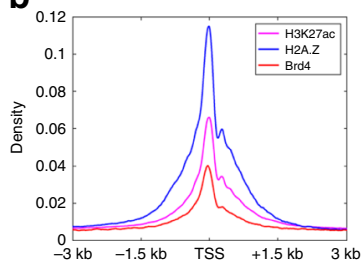

C

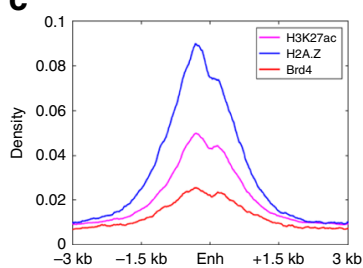

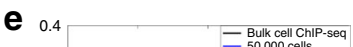

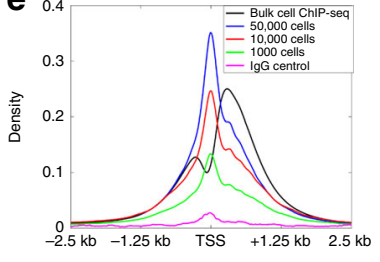

Fig. 1 ACT-seq robustly maps epigenetic marks in bulk-cell samples. a Genome browser image depicting enrichment of the indicated epigenetic factors in HEK293T cells at a representative genomic region. Data were obtained using ACT-seq (blue, green) or ChIP-seq (red). The ChIP-seq samples were obtained from published ENCODE data sets. A mock IgG sample (aggregated from all ACT-seq IgG replicates) is included as a comparative control for enrichment. b Metagene profile of average H3K27ac, H2A.Z, and Brd4 enrichment at the transcription start site (TSS) region of annotated genes from the hg19 genome. c Metagene profile of average H3K27ac, H2A.Z, and Brd4 enrichment at enhancer (Enh) regions. Enhancers were identified as regions enriched for H3K27ac that did not overlap with an annotated TSS. d Genome browser image depicting enrichment of H3K4me3 in HEK293T samples of the indicated cell number at a representative genomic region. A published ChIP-seq sample from ENCODE is provided for comparison. e Metagene profile of average H3K4me3 enrichment at the TSS region of annotated genes from the hg19 genome. Samples were obtained using the indicated number of cells. A published ChIP-seq sample from ENCODE is provided for comparison

(4.3\%). This small number of potential doublets is unlikely to perturb biological interpretation of the data. However, the possibility of a small number of residual cell doublets should be considered by investigators if their samples are extremely heterogenous or if the expected number of cells to be sequenced is very small. The total time required for an ACT-seq experiment is $5-6 \mathrm{~h}$, which is sufficient to generate libraries for a dozen epigenetic features. This duration is much shorter compared to other commonly used methods for mapping histone modifications. Similarly, an iACT-seq experiment requires only one day of bench work to construct libraries for thousands of single cells. Based on these attributes and its ease of use, we conclude that ACT-seq presents an attractive alternative to existing techniques for profiling epigenetic marks in bulk and single-cell samples.

\section{Methods}

Detailed protocols. The ACT-seq and iACT-seq methods, including variants using formaldehyde crosslinking, are available in bullet-point format at The Protocol Exchange website [https://protocolexchange.researchsquare.com/].

Preparation of the PA-Tnp transposon complex for bulk cells. The expression vector containing the PA-Tnp fusion construct is available from Addgene under accession number 121137 [http://www.addgene.org/121137/]. Recombinant PATnp protein was expressed in BL21-Gold(DE3) competent cells (Agilent cat \# 230132) and purified using nickel beads. Quality and purity of the isolated protein was assayed using SDS-PAGE. In all, $10 \mu \mathrm{M}$ of recombinant PA-Tnp protein was incubated for $10 \mathrm{~min}$ at $25^{\circ} \mathrm{C}$ in complex formation buffer $(50 \mathrm{mM}$ Tris $\mathrm{pH} 8.0$, $150 \mathrm{mM} \mathrm{NaCl}, 0.05 \%$ Triton X-100, $12.5 \%$ glycerol) containing $50 \mu \mathrm{M}$ of $5^{\prime}$ complex barcode and $50 \mu \mathrm{M}$ of $3^{\prime}$ complex barcode. $1 \mu \mathrm{L}$ of the appropriate antibody (at the stock concentration provided by the manufacturers) was added to $12 \mu \mathrm{L}$ of prepared complex and incubated at $25^{\circ} \mathrm{C}$ for $60 \mathrm{~min}$. The antibodies used in this study, listed with the manufacturer's provided concentration information, were: anti-H3K4me3 (Millipore cat \# 17-614, indicated $3 \mu \mathrm{L}$ for one ChIP sample), anti-H3K4me2 (Abcam cat \# ab32356, 0.1-0.5 mg/mL), anti-H3K4me1 (Abcam ab8895, <1 mg/mL), anti-H3K27ac (Abcam ab4729, <1 mg/mL), anti-H2A.Z (Abcam ab4174, 0.8 to $1 \mathrm{mg} / \mathrm{mL}$ ), anti-BRD4 (Bethyl cat \# A301-985A100, $1 \mathrm{mg} / \mathrm{mL}$ ), and normal IgGs (Millipore cat \# cs200581, $1 \mathrm{mg} / \mathrm{mL}$ ).

Bulk-cell chromatin binding and tagmentation. HEK293T cells were permeabilized using a 10-min incubation on ice in $500 \mu \mathrm{L}$ of complex formation buffer per $\sim 1$ million cells. After permeabilization, all subsequent centrifugations of cells were performed using the following procedure: $1 \mathrm{~min}$ spin at $250 \times \mathrm{g}$, rotate tubes $180^{\circ}$, repeat spin, leave $\sim 10 \mu \mathrm{L}$ of solution in the tube when removing buffer. Pelleted cells were washed once with $500 \mu \mathrm{L}$ of complex buffer and suspended in another $500 \mu \mathrm{L}$ of buffer. Aliquots of 1000 cell equivalents were transferred volumetrically to clean microcentrifuge tubes and adjusted to $50 \mu \mathrm{L}$ total volume using complex buffer. In total, $5 \mu \mathrm{L}$ of antibody-bound PA-Tnp complex was added to each $50 \mu \mathrm{L}$ cell aliquot and incubated at room temperature for $60 \mathrm{~min}$ to allow chromatin binding. Unbound complex was removed using three washes performed as follows: pellet and suspend cells in $500 \mu \mathrm{L}$ of wash buffer $(50 \mathrm{mM}$ Tris $\mathrm{pH} 8.0$, $150 \mathrm{mM} \mathrm{NaCl}, 0.1 \%$ Triton X-100), rotate tube for $5 \mathrm{~min}$ at room temperature, repeat. Pelleted cells were rinsed with $500 \mu \mathrm{L}$ of rinse buffer $(50 \mathrm{mM}$ Tris $\mathrm{pH} 8.0$, $50 \mathrm{mM} \mathrm{NaCl}, 0.1 \%$ Triton X-100). The rinse buffer was removed and $90 \mu \mathrm{L}$ of reaction buffer $\left(50 \mathrm{mM}\right.$ Tris $\mathrm{pH} 8.0,150 \mathrm{mM} \mathrm{NaCl}, 10 \mathrm{mM} \mathrm{MgCl}_{2}, 0.1 \%$ Triton $\mathrm{X}-100$ ) was added to the pelleted cells for a total volume of $\sim 100 \mu \mathrm{L}$. The tubes were incubated for $30 \mathrm{~min}$ at $37^{\circ} \mathrm{C}$ to allow transposition to occur. The reaction was terminated by addition of $4 \mu \mathrm{L}$ of $0.5 \mathrm{M}$ EDTA, $2 \mu \mathrm{L}$ of $10 \%$ SDS, and $1 \mu \mathrm{L}$ of $20 \mathrm{mg} / \mathrm{mL}$ Proteinase $\mathrm{K}$ followed by a $60 \mathrm{~min}$ incubation at $55^{\circ} \mathrm{C}$.

DNA was purified using phenol-chloroform extraction and ethanol precipitation. The DNA pellet was suspended in $10 \mu \mathrm{L}$ of $10 \mathrm{mM}$ Tris $\mathrm{pH}$ 8.0. PCR reactions for library preparation were performed by adding the following to the $10 \mu \mathrm{L}$ DNA sample: $25 \mu \mathrm{L}$ of Phusion High-fidelity PCR Master Mix (NEB catalog \# M0531S), $1 \mu \mathrm{L}$ of $5^{\prime}$ library index barcode, $1 \mu \mathrm{L}$ of $3^{\prime}$ library index barcode, and $13 \mu \mathrm{L}$ of nuclease-free water. Amplification was performed using an initial step of $72^{\circ} \mathrm{C}$ for $5 \mathrm{~min}$ followed by 15 cycles of: $98^{\circ} \mathrm{C}$ for $10 \mathrm{~s}, 65^{\circ} \mathrm{C}$ for $30 \mathrm{~s}, 72^{\circ} \mathrm{C}$ for $15 \mathrm{~s}$, and a final extension step of $72^{\circ} \mathrm{C}$ for $5 \mathrm{~min}$. The PCR products were analyzed using agarose gel electrophoresis. Fragments of the desired size were excised and purified using a QIAquick Gel Extraction kit (Qiagen cat \# 28506). 
a

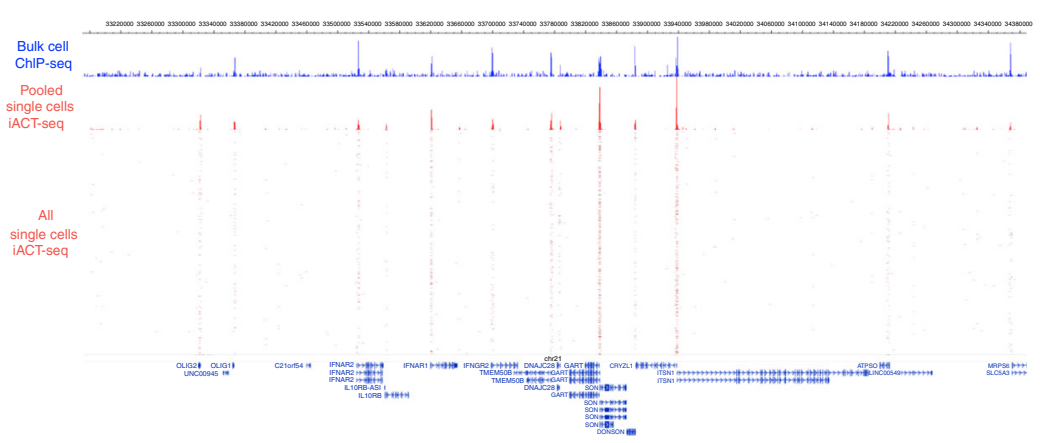

C

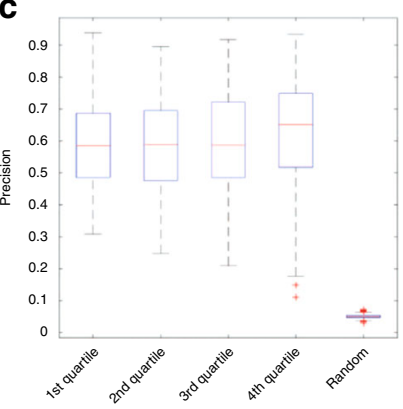

d

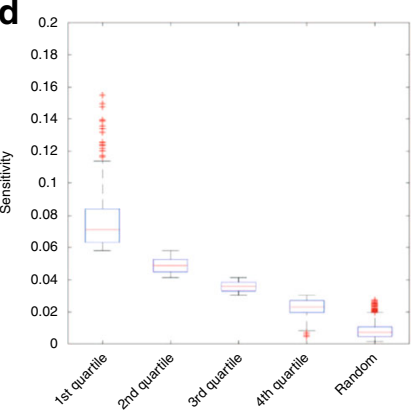

b

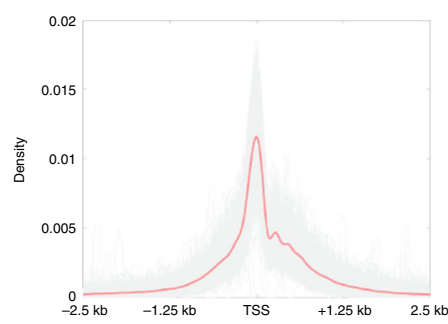

Fig. 2 ACT-seq reproducibly maps epigenetic marks in single cells. a Genome browser image of H3K4me3 peaks from bulk-cell ChIP-seq (blue) and pooled iACT-seq (red). The mapped reads from all 1246 individual cells are plotted below the aggregate peaks. Each row represents a single cell. $\mathbf{b}$ Metagene profile of H3K4me3 enrichment at the TSS region of genes from the hg18 genome for all single cells. The red line indicates average enrichment for all single cells from the iACT-seq data set. c, d Precision and sensitivity plots for the H3K4me3 scACT-seq data set. These values were calculated in the same manner as was done previously ${ }^{16}$. Data are divided into quartiles with the central marks indicating the median values. The bottom and top edges of the boxes indicate the 25th and 75th percentiles, respectively. The whiskers indicate the boundaries of the data. e Scatter plots depicting the correlation in H3K4me3 peak enrichment in counts per million (CPM) between ENCODE bulk-cell ChIP-seq data ( $x$-axis) and pooled scACT-seq data ( $y$-axes). Peaks identified as enriched using both the ChIP-seq and scACT-seq methods were included. $\mathbf{f}$ Venn diagram indicating the numbers of significantly enriched H3K4me3 peaks with at least $1 \mathrm{bp}$ overlap between a bulk-cell ENCODE ChIP-seq data set (blue) and pooled scACT-seq data (red)

Preparation of PA-Tnp for single cells. Preparation of the PA-Tnp complex was performed in PCR tube strips totaling 96 wells, with each well receiving a unique combination of $5^{\prime}$ and $3^{\prime}$ complex barcodes. The following were mixed in each well: $1 \mu \mathrm{L}$ of $1 \mu \mathrm{g} / \mu \mathrm{L}$ recombinant PA-Tnp protein, $0.75 \mu \mathrm{L}$ of $10 \mathrm{mM} 5^{\prime}$ complex barcode, $0.75 \mu \mathrm{L}$ of $10 \mathrm{mM} 3^{\prime}$ complex barcode, and $2.5 \mu \mathrm{L}$ of $2 \mathrm{X}$ complex buffer. Tube strips were incubated at $25^{\circ} \mathrm{C}$ for $10 \mathrm{~min}$ to allow complex formation. In all, $1 \mu \mathrm{L}$ of each of the 96 prepared PA-Tnp complexes were transferred to 96 fresh PCR tubes, carefully mixed with $0.8 \mu \mathrm{L}$ of the desired antibody, and incubated at room temperature for $60 \mathrm{~min}$.

Single-cell chromatin binding and tagmentation. One million HEK293T cells (ATCC accession number CRL-3216) were washed and permeabilized as described above. Aliquots of 10,000 permeabilized cells were volumetrically dispensed into the wells. The samples were mixed and incubated at room temperature for $60 \mathrm{~min}$ to allow chromatin binding. Unbound complex was removed by centrifuging the PCR tube strips for $3 \mathrm{~min}$ at $250 \times g$ and carefully discarding all but $\sim 10 \mu \mathrm{L}$ of the solutions. In total, $50 \mu \mathrm{L}$ of complex buffer was added to each well, and the centrifugation and volume removal steps were repeated. The cell solutions were suspended and pooled together in a single microcentrifuge tube for a total of $\sim 1 \mathrm{~mL}$ of combined volume. The tube containing the pooled cells was centrifuged for $1 \mathrm{~min}$ at $250 \times g$, rotated $180^{\circ}$, centrifuged again, and all but $\sim 20 \mu \mathrm{L}$ of solution was carefully removed. The cells were suspended in $200 \mu \mathrm{L}$ of complex buffer and filtered using a cell strainer test tube (Corning cat \# 352235). A FACS instrument was used to distribute the (un-stained) cells into 96 clean wells containing 18 cells each. The transposition reaction was initiated by adding $10 \mathrm{mM} \mathrm{MgCl}_{2}$ to each well in $10 \mu \mathrm{L}$ final volume followed by a $37-{ }^{\circ} \mathrm{C}$ incubation for $60 \mathrm{~min}$. The reactions were terminated by addition of $12 \mathrm{mM}$ EDTA, $0.1 \% \mathrm{SDS}$, and $1 \mu \mathrm{L}$ of $1 \mathrm{AU} / \mathrm{mL}$ protease (Qiagen cat \# 19157) to each well followed by a 60-min incubation at $55^{\circ} \mathrm{C}$. Each sample was transferred to a separate microcentrifuge tube and subjected to phenol-chloroform extraction followed by ethanol precipitation to purify the DNA. The samples were suspended in $10 \mu \mathrm{L}$ each of $10 \mathrm{mM}$ Tris $\mathrm{pH} 8.0$ and transferred back to PCR tube strips.

For library preparation, $0.5 \mu \mathrm{L}$ of $10 \mathrm{mM} \mathrm{3}$ ' library index barcode was added to each of the 96 wells, with each well receiving a $3^{\prime}$ barcode with a unique sequence. Each well then received $10 \mu \mathrm{L}$ of Phusion High-fidelity PCR Master Mix and $0.5 \mu \mathrm{L}$ of $10 \mathrm{mM}$ universal $5^{\prime}$ library index barcode. Eighteen cycles of PCR amplification were performed using the program described above. In total, $10 \mu \mathrm{L}$ of each PCR product were pooled and purified using three columns of a MinElute PCR Purification Kit (Qiagen cat \# 28004) for a total elution volume of $30 \mu \mathrm{L}$. Fragments of the desired size were gel-purified as described above.

Sequencing and data analysis. Paired-end sequencing was performed using an Illumina HiSeq 2500 platform. The resulting reads were mapped to the hg18 reference genome using Bowtie $2^{18}$. Data analysis and visualization was performed using custom $\mathrm{R}$ and Matlab scripts.

The TSS density profiles were computed using HOMER ${ }^{19}$. H3K4me3 peaks were identified using SICER ${ }^{20}$ with following parameters: gap size $=200 \mathrm{bp}$ and window size $=200 \mathrm{bp}$. The function "findOverlaps" from the R package GenomicRanges ${ }^{21}$ was used to compute the overlap between two sets of peaks. Spearman correlation was used to compare the read density between libraries. The ENCODE ChIP-seq data sets used for comparison were downloaded from GEO under accession numbers GSM2711409 (H3K27ac), GSM2711410 (H3K4me1), GSM2711411 (H3K4me2), and GSM945288 (H3K4me3)).

To filter out potential doublets from our iACT-seq sample population, we first calculated the collision rate (the predicted percentage of cell barcodes that corresponded to doublets) as described previously ${ }^{15}$. Chen et al. ${ }^{22}$ determined that doublets contain a higher read density than single cells. Using their approach, we filtered out the top $\sim 8 \%$ of cells from each well based on our calculated collision rate. We also removed low-quality libraries with fewer than 500 reads. After trimming, 1246 single-cell libraries remained. To estimate the number of doublets remaining after filtering, we performed a simulation as described in Supplementary File 1. Based on this simulation, we estimated that 54 doublets remained in the cell population. Mapping statistics are included in Supplementary Data 1 and summarized in Supplementary Fig. 6.

Oligonucleotide sequences. The names and sequences of oligonucleotides used in this study are available in Excel format in Supplementary Data 2. For background information on how these oligos were designed, please see the Illumina Adapter Sequences document at the Illumina Support Center website [https://support.illumina. com/downloads/illumina-adapter-sequences-document-1000000002694.html]. 
Reporting summary. Further information on research design is available in the Nature Research Reporting Summary linked to this article.

\section{Data availability}

Next-generation sequencing data are available at the Gene Expression Omnibus under accession number GSE125971. All other data are available from the authors upon reasonable request.

\section{Code availability}

The custom script used to estimate the percentage of doublet samples removed from the single-cell data is provided as Supplementary Software. Code for any part of the data analysis is freely available upon request.

Received: 17 January 2019 Accepted: 18 July 2019

Published online: 20 August 2019

\section{References}

1. Lo, P.-K. \& Zhou, Q. Emerging techniques in single-cell epigenomics and their applications to cancer research. J. Clin. Genom. 1, https://doi.org/10.4172/ JCG.1000103 (2018).

2. Picelli, S. Single-cell RNA-sequencing: the future of genome biology is now. RNA Biol. 14, 637-650 (2016).

3. Clark, S. J., Lee, H. J., Smallwood, S. A., Kelsey, G. \& Reik, W. Single-cell epigenomics: powerful new methods for understanding gene regulation and cell identity. Genome Biol. 17, 72-72 (2016).

4. Lawrence, M., Daujat, S. \& Schneider, R. Lateral thinking: how histone modifications regulate gene expression. Trends Genet.: TIG 32, 42-56 (2016).

5. Lai, B. et al. Trac-looping measures genome structure and chromatin accessibility. Nat. Methods 15, 741-747 (2018).

6. Buenrostro, J. D., Giresi, P. G., Zaba, L. C., Chang, H. Y. \& Greenleaf, W. J. Transposition of native chromatin for fast and sensitive epigenomic profiling of open chromatin, DNA-binding proteins and nucleosome position. Nat. Methods 10, 1213-1218 (2013).

7. Buenrostro, J. D. et al. Single-cell chromatin accessibility reveals principles of regulatory variation. Nature 523, 486-490 (2015).

8. Thurman, R. E. et al. The accessible chromatin landscape of the human genome. Nature 489, 75-82 (2012).

9. Morgan, M. A. J. et al. A cryptic Tudor domain links BRWD2/PHIP to COMPASS-mediated histone H3K4 methylation. Genes Dev. 31, 2003-2014 (2017).

10. Creyghton, M. P. et al. Histone H3K27ac separates active from poised enhancers and predicts developmental state. Proc. Natl Acad. Sci. USA 107, 21931-21936 (2010).

11. Barski, A. et al. High-resolution profiling of histone methylations in the human genome. Cell 129, 823-837 (2007).

12. Zhang, W. et al. Bromodomain-containing protein 4 (BRD4) regulates RNA polymerase II serine 2 phosphorylation in human CD4+ T cells. J. Biol. Chem. 287, 43137-43155 (2012).

13. Zheng, Q. et al. Reversible histone glycation is associated with disease-related changes in chromatin architecture. Nat. Commun. 10, 1289 (2019).

14. Rosenberg, A. B. et al. Single-cell profiling of the developing mouse brain and spinal cord with split-pool barcoding. Science 360, 176-182 (2018).

15. Cusanovich, D. A. et al. Multiplex single cell profiling of chromatin accessibility by combinatorial cellular indexing. Science 348, 910-914 (2015).

16. Rotem, A. et al. Single-cell ChIP-seq reveals cell subpopulations defined by chromatin state. Nat. Biotechnol. 33, 1165-1172 (2015).

17. Harada, A. et al. A chromatin integration labelling method enables epigenomic profiling with lower input. Nat. Cell Biol. https://doi.org/10.1038/ s41556-018-0248-3 (2018).
18. Langmead, B. \& Salzberg, S. L. Fast gapped-read alignment with Bowtie 2. Nat Methods 9, 357-359 (2012).

19. Heinz, S. et al. Simple combinations of lineage-determining transcription factors prime cis-regulatory elements required for macrophage and B cell identities. Mol. Cell 38, 576-589 (2010).

20. Xu, S., Grullon, S., Ge, K. \& Peng, W. Spatial clustering for identification of ChIP-enriched regions (SICER) to map regions of histone methylation patterns in embryonic stem cells. Methods Mol. Biol. 1150, 97-111 (2014).

21. Lawrence, M. et al. Software for computing and annotating genomic ranges. PLoS Comput. Biol. 9, e1003118 (2013).

22. Chen, X., Miragaia, R. J., Natarajan, K. N. \& Teichmann, S. A. A rapid and robust method for single cell chromatin accessibility profiling. Nat. Commun. 9, 5345 (2018).

\section{Acknowledgements}

We thank Dr. Kairong Cui for providing the HEK293T cells; the National Heart, Lung and Blood Institute DNA Sequencing Core Facility for sequencing the libraries; and the National Heart, Lung, and Blood Institute Flow Cytometry Core facility for sorting the cells. The work was supported by Division of Intramural Research, National Heart, Lung and Blood Institute.

\section{Author contributions}

B.C., K.Z., and W.L.K. participated in writing the manuscript. K.Z., B.C., Q.T., and J.Y.K. designed the experiments and performed the bench work. W.L.K. and B.C. performed the bioinformatics analyses and figure generation. Q.T., K.Z., G.H., and J.P. designed the oligonucleotides indexes for the iACT-seq barcoding strategy.

\section{Additional information}

Supplementary Information accompanies this paper at https://doi.org/10.1038/s41467 019-11559-1.

Competing interests: The authors declare no competing interests.

Reprints and permission information is available online at http://npg.nature.com/ reprintsandpermissions/

Peer review information: Nature Communications thanks Benjamin Schuster-Böckler and the other anonymous reviewer(s) for their contribution to the peer review of this work.

Publisher's note: Springer Nature remains neutral with regard to jurisdictional claims in published maps and institutional affiliations.

Open Access This article is licensed under a Creative Common Attribution 4.0 International License, which permits use, sharing, adaptation, distribution and reproduction in any medium or format, as long as you give appropriate credit to the original author(s) and the source, provide a link to the Creative Commons license, and indicate if changes were made. The images or other third party material in this article are included in the article's Creative Commons license, unless indicated otherwise in a credit line to the material. If material is not included in the article's Creative Commons license and your intended use is not permitted by statutory regulation or exceeds the permitted use, you will need to obtain permission directly from the copyright holder. To view a copy of this license, visit http://creativecommons.org/ licenses/by/4.0/.

This is a U.S. Government work and not under copyright protection in the US; foreign copyright protection may apply 2019 